\title{
Exact solution of inflationary model with minimum length
}

\author{
Achim Kempf \\ Department of Applied Mathematics, University of Waterloo \\ Waterloo, Ontario, N2L 3G1, Canada \\ Larissa Lorenz \\ Institut d'Astrophysique de Paris, 98bis boulevard Arago \\ 75014 Paris, France
}

\begin{abstract}
Within the inflationary scenario, Planck scale physics should have affected the comoving modes' initial conditions and early evolution, thereby potentially affecting the inflationary predictions for the cosmic microwave background (CMB). This issue has been studied extensively on the basis of various models for how quantum field theory (QFT) is modified and finally breaks down towards the Planck scale. In one model, in particular, an ultraviolet cutoff was implemented into QFT through generalized uncertainty relations which have been motivated from general quantum gravity arguments and from string theory. Here, we improve upon prior numerical and semi-analytical results by presenting the exact mode solutions for both de Sitter and power-law inflation in this model. This provides an explicit map from the modes' initial conditions, which are presumably set by quantum gravity, to the modes' amplitudes at horizon crossing and thus to the inflationary predictions for the CMB. The solutions' particular behaviour close to the cutoff scale suggests unexpected possibilities for how the degrees of freedom of QFT emerge from the Planck scale.
\end{abstract}




\section{Introduction}

Within the inflationary scenario, spacetime typically inflated to the extent that comoving modes which are of cosmological size today originated well beyond the Planck scale (and any other natural ultraviolet (UV) cutoff scale such as the string scale). This observation has led to a growing body of work exploring the tantalizing prospect that the predictions of inflation could be observably affected by quantum gravity. For early papers on this subject see, e.g., [1, 2, 3, 4, 5].

Technically, all possible Planck scale effects on the comoving modes, $k$, at late times take the form of a nontrivial $k$-dependent selection of solution to the ordinary mode equation, see, e.g., [6. This is because, independent of the details of Planck scale physics, each mode's evolution equation reduces to the ordinary wave equation as soon as its proper wave length is significantly larger than the Planck scale. It has been possible, therefore, to determine on these general grounds that quantum gravity effects should manifest themselves qualitatively in the form of superimposed oscillations in the CMB spectra as well as in a breaking of the consistency relation between the scalar/tensor ratio and the tensor spectral index of single field inflation, see, e.g., 7, 8, 9, 10, 11].

Any quantitative predictions, however, are particular to each particular theory of quantum gravity. Concretely, for wavelengths closer and closer to the cutoff scale the framework of QFT should hold - with characteristic increasing corrections - until it finally breaks down at the cutoff scale. A theory of quantum gravity, be it, e.g., loop quantum gravity or $M$ theory, should eventually allow one to calculate those characteristic corrections to the framework of QFT in this "sub-Planckian" regime, i.e., in the regime of wavelengths larger than but close to the cutoff length. In addition, the theory of quantum gravity should allow one to determine the initial condition for the evolution of a comoving mode within the framework of QFT, namely at the time when the mode's proper wavelength first exceeds the cutoff length.

In this context, first-principle calculations are very difficult, of course. Therefore, a number of authors have modelled the corrections to the framework of QFT from quantum gravity simply as UV modifications to the dispersion relations. The implied effects on the inflationary predictions for the CMB have been investigated, for example, in 1, 2, 3, 12. Particular modifications to the dispersion relations for short wavelengths have been motivated either ad hoc or on the basis of analogies to the propagation of waves in condensed matter systems. It was found that the effects of Planck scale physics could be considerable, even though there appear to be strong constraints due to a possibly strong backreaction problem, see, e.g., 13 .

A less ad hoc approach 4 4 has been to model the behaviour of quantum field theory in the subPlanckian regime through the implementation of corrections to the first quantization uncertainty relations, see 14. These corrections implement an ultraviolet cutoff in the form of a finite minimum uncertainty in spatial distances, which has been motivated by studies in quantum gravity and string theory, see, e.g., 15, 16, 17, 18, 19.

This approach to modelling Planck scale physics in inflation was further investigated in particular in [5, $7,9,10$. So far it has been possible to solve only approximations of the mode equation that arises in this approach. In fact, to solve the exact mode equation is known to be highly nontrivial even numerically because of a particular singular behaviour of the mode equation at the mode's starting time. Here, we are following up on this series of papers.

In particular, we calculate here the exact solutions to the exact mode equation in both the de Sitter and power-law backgrounds. This allows us then to follow the mode solutions back towards the time when the proper wavelength approaches the cutoff length. That in turn allows us to calculate the exact behaviour of physical quantities such as the field fluctuation amplitudes and the Hamiltonian towards the Planckian regime. We obtain, therefore, a fully explicit model in which to explore possible mechanisms that could impose initial conditions on comoving modes as they enter into the regime of validity of quantum field theory.

\section{Short distance physics and in- flation}

\subsection{UV cutoff through minimum length uncertainty}

A rather general assumption about quantum field theory in the sub-Planckian regime is that it should still possess for each coordinate a linear operator $\mathrm{x}^{i}$ (inherited from first quantization) whose formal expectation values (e.g., in the space of fields that are being summed over in the path integral) are real. The $\mathrm{x}^{i}$ may or may not commute. As shown in 14, this implies that the short distance structure of any such coordinate, considered separately, can only be continuous, discrete, or "unsharp" in one of two particular ways. Studies in quantum gravity and string theory point towards one of these two unsharp cases, 
namely the case of coordinates $\mathbf{x}$ whose formal uncertainty $\Delta \mathbf{x}$ possesses a finite lower bound $\Delta \mathbf{x}_{\min }$ at the Planck or string scale. This short distance structure arises from quantum gravity correction terms to the uncertainty relation (see [20]):

$$
\Delta x \Delta p \geq \frac{1}{2}\left(1+\beta(\Delta p)^{2}+\ldots\right)
$$

Here, $\beta$ is a positive constant which, as is easily verified, parametrizes the cutoff length through $\Delta x_{\min }=$ $\sqrt{\beta}$. The units are such that $\hbar=1$. As was first pointed out in 21, uncertainty relations of this form arise from corrections to the canonical commutation relations which, for example in the case of one space dimension, take this form:

$$
[\mathbf{x}, \mathbf{p}]=i\left(1+\beta \mathbf{p}^{2}+\ldots\right)
$$

It was shown in 22 that the multi-dimensional generalization is unique to first order in $\beta$ if rotational and translational symmetry is to be preserved.

Let us remark that, as was shown in 23], this type of natural UV cutoff implies that physical fields possess a finite bandwidth in the information theoretic sense: In the presence of this short distance structure one can apply Shannon's sampling theorem to conclude that if the values of a physical field are known on any set of points with average density above twice the Planck density then the values of the field everywhere are already determined and can be explicitly calculated from these samples ${ }^{1}$. Notice that no sampling lattice is preferred, as all sampling lattices of sufficiently high average density can be used to reconstruct the fields. Therefore, in a theory with this type of natural cutoff spacetime can be viewed as continuous, in which case the conservation of the spatial symmetries is displayed, while, fully equivalently, in the same theory spacetime can also be viewed as discrete, in which case the theory's UV finiteness is displayed. So far, this type of natural UV cutoff has been considered mostly within models that break Lorentz invariance, as we will here. The generalization to a generally covariant setting has been started in [24].

\subsection{Mode generation in the presence of the UV cutoff}

In 4], the short distance cutoff formulated in (2) was applied to the theory of a minimally coupled massless

\footnotetext{
${ }^{1}$ The situation is mathematically identical, for example, to that of HiFi music signals of bandwidth $20 \mathrm{KHz}$, whose amplitude samples are recorded on CDs at a rate of $4 \cdot 10^{4}$ samples per second. Using Shannon's theorem, CD players are able to precisely reconstruct the continuous music signal at all time from these discrete samples.
}

real scalar field $\phi(\mathbf{x}, t)$ in an expanding FriedmannRobertson-Walker (FRW) background. This setup can be used to describe the quantum dynamics of the tensor as well as the scalar fluctuations in inflation. Concerning the mode evolution, the only difference is that, in the latter case, the scale factor $a$ in the mode equation is replaced by $z=\phi_{0}^{\prime} a^{2} / a^{\prime}$. Here, $\phi_{0}$ is the bulk zero mode of the inflaton field with the prime denoting differentiation with respect to $\eta$. (It should be kept in mind, however, that the Hamiltonian of the gravitational waves that are due to tensor modes contributes to the energy momentum tensor only from second order.)

The approach of 4] was continued in [5] as well as in a series of papers by Easther et al. [7, 9, 10. In each case, for simplicity, the notation for the tensor modes was used (i.e. with the mode equation containing $a$ instead of $z$ ), as we will also do here.

We assume the case of spatial flatness of the background spacetime and we use the conformal time coordinate $\eta$. Note that the UV cutoff $\Delta x_{\min }=\sqrt{\beta}$ is introduced in proper distances as opposed to comoving distances. The action for the field $\phi(x, \eta)$ then reads, see 4]:

$$
\begin{aligned}
S= & \int d \eta d^{3} x \frac{1}{2 a}\left\{\left[\left(\partial_{\eta}+\frac{a^{\prime}}{a} \sum_{i=1}^{3} \partial_{x^{i}} x^{i}-\frac{3 a^{\prime}}{a}\right) \phi\right]^{2}\right. \\
& \left.-a^{2} \sum_{i=1}^{3}\left(\partial_{x^{i}} \phi\right)^{2}\right\}
\end{aligned}
$$

Note that our function $\phi_{\tilde{k}}(\eta)$ is related to $u_{\tilde{k}}$ in 7 by $u_{\tilde{k}}=1 / a^{2} \cdot \phi_{\tilde{k}}(\eta)$ and that our function $\nu(\eta, \tilde{k})$ is the function $\nu(\eta, \rho)$ from 7 multiplied by $a^{4}$. Using the operators

$$
\begin{gathered}
\mathbf{x}^{i}: \phi(x, \eta) \rightarrow x_{i} \phi(x, \eta) \\
\mathbf{p}^{i}: \phi(x, \eta) \rightarrow-i \partial / \partial x_{i} \phi(x, \eta)
\end{gathered}
$$

the action can be expressed representation independently in terms of these operators and the usual $L^{2}$ inner product of fields. While the fields' expression for the action (3) is held fixed the underlying commutation relations are now corrected to introduce the desired cutoff (2). The modification of the short distance behaviour then manifests itself whenever one writes the action in any representation, such as in comoving momentum space. For example, at distances close to the cutoff length, comoving modes no longer decouple. However, it is then still possible to find variables $(\eta, \tilde{k})$ in which the $\tilde{k}$ modes of the fields' Fourier transform do decouple ${ }^{2}$. The de-

\footnotetext{
${ }^{2}$ Note that, as discussed in 4 and $[5$, these variables $\tilde{k}$ approximate the comoving momentum variables $k$ only at long wavelengths.
} 
coupling modes are important as they describe the truly independent degrees of freedom. They will be denoted as $\phi_{\tilde{k}}(\eta)$. The realness of the field $\phi(\mathbf{x}, \eta)$ translates into $\phi_{\tilde{k}}^{*}=\phi_{-\tilde{k}}$. The action (3) can then be written in the form

$$
S=\int d \eta \int_{\tilde{k}^{2}<a^{2} / e \beta} d^{3} \tilde{k} \mathscr{L}
$$

with the Lagrangian

$$
\mathscr{L}=\frac{1}{2} \nu\left\{\left|\left(\partial_{\eta}-3 \frac{a^{\prime}}{a}\right) \phi_{\tilde{k}}(\eta)\right|^{2}-\mu\left|\phi_{\tilde{k}}(\eta)\right|^{2}\right\},
$$

where the coefficient functions are given by

$$
\begin{aligned}
\mu(\eta, \tilde{k}) & :=-\frac{a^{2} \operatorname{plog}\left(-\beta \tilde{k}^{2} / a^{2}\right)}{\beta\left[1+\operatorname{plog}\left(-\beta \tilde{k}^{2} / a^{2}\right)\right]^{2}}, \\
\nu(\eta, \tilde{k}) & :=\frac{\exp \left[-\frac{3}{2} \operatorname{plog}\left(-\beta \tilde{k}^{2} / a^{2}\right)\right]}{a^{4}\left[1+\operatorname{plog}\left(-\beta \tilde{k}^{2} / a^{2}\right)\right]} .
\end{aligned}
$$

The function plog used here, the "product log", is defined as the inverse of the function $x \rightarrow x e^{x}$ (also known as the Lambert $\mathrm{W}$-function). It will be important later that, at $x=-1 / e$, this function possesses an essential singularity.

Equally important will be the way in which the short distance cutoff ${ }^{3}$ affects the integration region of the action functional (4): each mode $\tilde{k}$ enters the action once $a(\eta)$ has grown enough so that the condition $\tilde{k}^{2}<a^{2} / e \beta$ holds, i.e., when:

$$
a\left(\eta_{c}\right)=\tilde{k} \sqrt{e \beta} \approx \tilde{k} \Delta x_{m i n} .
$$

Correspondingly, the mode equation that follows from the action (5), see 4,

$$
\phi_{\tilde{k}}^{\prime \prime}+\frac{\nu^{\prime}}{\nu} \phi_{\tilde{k}}^{\prime}+\left[\mu-3\left(\frac{a^{\prime}}{a}\right)^{\prime}-9\left(\frac{a^{\prime}}{a}\right)^{2}-3 \frac{a^{\prime} \nu^{\prime}}{a \nu}\right] \phi_{\tilde{k}}=0
$$

is an equation of motion which possesses a starting time. As had to be expected, the implementation of a UV cutoff in an expanding spacetime has led us to the problem of setting initial conditions on these newly emerging comoving modes. Before we solve the mode equation to obtain the solutions' early time behavior, let us briefly consider the mode solutions' late time behaviour.

\footnotetext{
${ }^{3}$ For $\beta \rightarrow 0,5$ reduces to the standard action. Note that Fourier transforming and scaling only commute up to a scaling factor of $a^{3}$.
}

\subsection{Late time behaviour}

At late times, when a mode's proper wavelength far exceeds the cutoff length, the mode becomes insensitive to the nontrivial short distance structure of spacetime. The modified mode equation (8) then reduces to the mode equation without cutoff, which (taking into account the non-commutativity of scaling and Fourier transforming) reads with our conventions $^{4}$ :

$$
\phi_{\tilde{k}}^{\prime \prime}-4 \frac{a^{\prime}}{a} \phi_{\tilde{k}}^{\prime}+\left(6\left(\frac{a^{\prime}}{a}\right)^{2}-3 \frac{a^{\prime \prime}}{a}+\tilde{k}^{2}\right) \phi_{\tilde{k}}=0
$$

Let us derive the late time behaviour of (9), namely $\eta \rightarrow 0$, in de Sitter space $a(\eta)=-1 / H \eta$. Since in this case we have $\frac{a^{\prime}}{a}=-\frac{1}{\eta}$ and $\frac{a^{\prime \prime}}{a}=\frac{2}{\eta^{2}}$, while $\tilde{k}^{2}$ is constant, equation (9) on large scales becomes:

$$
\phi_{\tilde{k}}^{\prime \prime}+4 \frac{1}{\eta} \phi_{\tilde{k}}^{\prime}=0
$$

The solution to this equation is given by

$$
\phi_{\tilde{k}}=B_{1}+\frac{B_{2}}{\eta^{3}} .
$$

So for late times, $\eta \rightarrow 0$, the mode function $\phi_{\tilde{k}}(\eta)$ diverges $\propto \frac{1}{\eta^{3}}$ in the de Sitter case. We recall that $\phi_{\tilde{k}}(\eta)=a^{3}(\eta) \Phi_{\tilde{k}}(\eta)$. Thus, as expected, the physical field's mode function $\Phi_{\tilde{k}}(\eta)$ approaches a constant for late times. Let us remark that the analogous calculation for classical fluctuations indicates that classical scalar or tensor fluctuations that existed before inflation would have their wavelength stretched but their amplitude maintained, i.e., they would not be "ironed out" by inflation. Inflation is usually thought to have lasted long enough so that, classically, the inflation-induced flattening of a fixed proper spatial volume would hinge on the assumption that there were no pre-existing spatial "ripples" down to wavelengths shorter than the Planck length. Of course, the Planck scale is expected to rule out the notion of pre-existing classical ripples that are shorter than the Planck length.

\section{The Hamiltonian}

Using the canonically conjugate field to $\phi_{\tilde{k}}$, see [4], $\pi_{\tilde{k}}(\eta)=\nu \phi_{-\tilde{k}}^{\prime}(\eta)-3 \nu \frac{a^{\prime}}{a} \phi_{-\tilde{k}}(\eta)$, the Hamiltonian corresponding to the action (3) takes the form

$H=\int_{\tilde{k}^{2}<\frac{a^{2}}{e \beta}} d^{3} \tilde{k}\left(\frac{1}{2 \nu} \pi_{\tilde{k}}^{*} \pi_{\tilde{k}}+\frac{1}{2} \nu \mu \phi_{\tilde{k}}^{*} \phi_{\tilde{k}}+3 \frac{a^{\prime}}{a} \pi_{\tilde{k}} \phi_{\tilde{k}}\right)$.

\footnotetext{
${ }^{4}$ Recall that at large scales we also have $\tilde{k} \rightarrow k$.
} 
To quantize one promotes $\hat{\phi}_{\tilde{k}}$ and $\hat{\pi}_{\tilde{k}}$ to operators satisfying the usual commutation relation $\left[\hat{\phi}_{\tilde{k}}, \hat{\pi}_{\tilde{r}}\right]=$ $i \delta^{3}(\tilde{k}-\tilde{r})$ in momentum space. Note that the field commutators are of course modified when expressed in position space.

In the Hamiltonian, the integration region increases over time, expressing that the field operators of comoving modes enter the Hamiltonian only when the mode's wavelength exceeds the cutoff length. This is significant because it means that the Hamiltonian contains operators which act nontrivially on the Hilbert space dimensions of a particular comoving mode $\tilde{k}$ only from that time $\eta_{c}(\tilde{k})$ when this mode's proper wavelength starts to exceed the cutoff length. In the literature, this time (which coincides with the essential singularity of the plog function) has been called the mode's "creation time" and we will stay with this terminology. Let us keep in mind, however, that the dimensions on which the harmonic oscillator of a given mode is represented are of course always in the Hilbert space. In the Heisenberg picture, all that happens at the creation time of a mode is that the mode's field and conjugate field operators enter the Hamiltonian for the first time, while conversely, in a shrinking universe, a mode's field operators would drop from the Hamiltonian when the mode's wavelength drops below the cutoff length.

We express the quantum field in terms of creation and annihilation operators $a_{\tilde{k}}^{\dagger}$ and $a_{\tilde{k}}$ and in terms of the mode functions $\phi_{\tilde{k}}(\eta)$ :

$$
\hat{\phi}_{\tilde{k}}(\eta)=a_{\tilde{k}} \phi_{\tilde{k}}(\eta)+a_{-\tilde{k}}^{\dagger} \phi_{-\tilde{k}}^{*}(\eta),
$$

Note that the notation $\phi_{\tilde{k}}(\eta)$ now no longer stands for the classical field (which obeys $\phi_{\tilde{k}}^{*}=\phi_{-k}$ ) but instead for a mode function. For the mode functions we can choose, as usual that $\phi_{\tilde{k}}=\phi_{|\tilde{k}|}$. The Wronskian condition

$$
\nu(\eta, \tilde{k})\left[\phi_{\tilde{k}}(\eta) \phi_{\tilde{k}}^{\prime *}(\eta)-\phi_{\tilde{k}}^{*}(\eta) \phi_{\tilde{k}}^{\prime}(\eta)\right]=i .
$$

insures that the so-defined quantum fields obey the canonical commutation relations. We can then rearrange the quantized Hamiltonian in the following form that we will later refer to:

$$
\begin{aligned}
\hat{H}= & \int_{\tilde{k}^{2}<\frac{a^{2}}{e \beta}} d^{3} \tilde{k}\left[\frac{\nu}{2}\left|\phi_{\tilde{k}}^{\prime}\right|^{2}+\frac{\nu}{2}\left(\mu-9\left(\frac{a^{\prime}}{a}\right)^{2}\right)\left|\phi_{\tilde{k}}\right|^{2}\right] \\
& \times\left(a_{\tilde{k}}^{\dagger} a_{\tilde{k}}+a_{-\tilde{k}}^{\dagger} a_{-\tilde{k}}\right) \\
+ & {\left[\frac{\nu}{2} \phi_{-\tilde{k}}^{\prime} \phi_{\tilde{k}}^{\prime}+\frac{\nu}{2}\left(\mu-9\left(\frac{a^{\prime}}{a}\right)^{2}\right) \phi_{-\tilde{k}} \phi_{\tilde{k}}\right] } \\
& \times\left(a_{\tilde{k}} a_{-\tilde{k}}\right) \\
+ & {\left[\frac{\nu}{2}\left(\phi_{-\tilde{k}}^{\prime} \phi_{\tilde{k}}^{\prime}\right)^{*}+\frac{\nu}{2}\left(\mu-9\left(\frac{a^{\prime}}{a}\right)^{2}\right)\left(\phi_{-\tilde{k}} \phi_{\tilde{k}}\right)^{*}\right] } \\
& \times\left(a_{\tilde{k}} a_{-\tilde{k}}\right)^{\dagger} \\
+ & {\left[\frac{\nu}{2}\left|\phi_{\tilde{k}}^{\prime}\right|^{2}+\frac{\nu}{2}\left(\mu-9\left(\frac{a^{\prime}}{a}\right)^{2}\right)\left|\phi_{\tilde{k}}\right|^{2}\right] \delta^{3}(0) . \quad(14) }
\end{aligned}
$$

To see that this Hamiltonian gives the correct equation of motion (8), it is straightforward to apply the Heisenberg equations and use the definition of $\hat{\pi}_{\tilde{k}}$. Note again the bound on the integral, which reflects the fact that each mode $\tilde{k}$ only contributes to the Hamiltonian from its creation time $\eta_{c}(\tilde{k})$ onwards; before that time, the operators $a_{\tilde{k}}$ and $a_{\tilde{k}}^{\dagger}$ are not contained in $\hat{H}$.

Let us note that, curiously, in the case of a shrinking universe the modes' creation and annihilation operators $a_{\tilde{k}}, a_{\tilde{k}}^{\dagger}$ successively drop out of the Hamiltonian. Thus, a growing number of states can no longer be "reached" by the Hamiltonian and the field operators while the universe is contracting. All information encoded in these states would no longer interact although it would become relevant again during a subsequent expanding phase.

We will be interested, in particular, in the ground state energy term of each comoving mode, given by the last line of (14):

$$
\begin{aligned}
\rho_{v a c, \tilde{k}}(\eta) & =\frac{\nu(\eta)}{2}\left|\phi_{\tilde{k}}^{\prime}(\eta)\right|^{2} \\
& +\frac{\nu(\eta)}{2}\left|\phi_{\tilde{k}}(\eta)\right|^{2}\left(\mu(\eta)-9\left(\frac{a^{\prime}(\eta)}{a(\eta)}\right)^{2}\right)
\end{aligned}
$$

Since the Hamiltonian is closely related to the 00 component of the energy momentum tensor $T^{\mu}{ }_{\nu}$, (15) essentially describes the energy contribution of each mode which arises when the mode outgrows the cutoff scale. Two time dependencies determine the evolution of the modes' ground state energy (15): first, the behaviour of the coefficients $\nu(\eta, \tilde{k})$ and $\mu(\eta, \tilde{k})$, and second the mode function $\phi_{\tilde{k}}(\eta)$. While the former are already known explicitly (see (6) and (7)), as we will discuss, the choice of solution to the equation 
of motion (8) for the mode function will nontrivially affect the ground state energy.

\section{Solving the mode equation}

We will now derive the exact solutions to (8) which obey the Wronskian condition (13). The difficulty here is due to the plog-functions in $\mu(\eta, \tilde{k})$ and $\nu(\eta, \tilde{k})$ : the equation of motion for each $\tilde{k}$-mode has an irregular singular point at the mode's creation time $\eta_{c}(\tilde{k})$. In previous work, 7], the mode equation was replaced by an approximate mode equation which had a regular singular point at the mode's creation time. The exact solutions to this approximate mode equation were obtained both as power series and in analytical form.

Our aim now is to obtain the exact solutions to the exact mode equation. To this end, we employ a suitable variable transformation that turns the irregular singular point into a regular singular point, without the need for approximations. We then apply the Frobenius method to derive the exact solutions, namely in terms of convergent power series and logarithms.

\subsection{Singularity transformation}

Technically, $\eta_{c}(\tilde{k})$ is called an irregular singular point of (8) because the term

$$
\left(\eta-\eta_{c}(\tilde{k})\right)^{2}\left[\mu-3\left(\frac{a^{\prime}}{a}\right)^{\prime}-9\left(\frac{a^{\prime}}{a}\right)^{2}-3 \frac{a^{\prime} \nu^{\prime}}{a \nu}\right]
$$

and the term

$$
\left(\eta-\eta_{c}(\tilde{k})\right) \cdot \frac{\nu^{\prime}}{\nu}
$$

do not have convergent Taylor expansions around this point. Let us now define a new variable $\tau$,

$$
\tau=\operatorname{plog}\left(-\beta \tilde{k}^{2} / a(\eta)^{2}\right),
$$

which plays the role of time, but is a function of both $\eta$ and $\tilde{k}$. The scale factor $a(\eta)$ and the coefficient functions $\nu(\eta, \tilde{k})$ and $\mu(\eta, \tilde{k})$ then $\operatorname{read}^{5}$

$$
\begin{aligned}
\tilde{a}(\tau) & :=\tilde{k} \sqrt{\frac{\beta}{-\tau e^{\tau}}}, \\
\tilde{\mu}(\tau, \tilde{k}) & :=\frac{\tilde{k}^{2}}{e^{\tau}(1+\tau)^{2}}, \\
\tilde{\nu}(\tau, \tilde{k}) & :=\frac{\tau^{2} e^{\frac{\tau}{2}}}{\beta^{2} \tilde{k}^{4}(1+\tau)} .
\end{aligned}
$$

It is straightforward to translate the derivatives $\partial / \partial \eta$ into derivatives in terms of derivatives $\partial / \partial \tau$ and we then obtain that the equation of motion (8) takes the form:

$$
\begin{aligned}
0=\tilde{\phi}_{\tilde{k}}^{\prime \prime} & +\left(\frac{\tilde{\nu}^{\prime}}{\tilde{\nu}}-\frac{\eta^{\prime \prime}}{\eta^{\prime}}\right) \tilde{\phi}_{\tilde{k}}^{\prime} \\
+ & {\left[\tilde{\mu} \eta^{\prime 2}-3\left(\frac{\tilde{a}^{\prime \prime}}{\tilde{a}}\right)-6\left(\frac{\tilde{a}^{\prime}}{\tilde{a}}\right)^{2}\right.} \\
& \left.-3 \frac{\tilde{a}^{\prime} \tilde{\nu}^{\prime}}{\tilde{a} \tilde{\nu}}+3 \frac{\tilde{a}^{\prime}}{\tilde{a}} \frac{\eta^{\prime \prime}}{\eta^{\prime}}\right] \tilde{\phi}_{\tilde{k}}
\end{aligned}
$$

Note that we now let the prime denote differentiation with respect to $\tau$ rather than $\eta$.

In order to make those expressions explicit in an example, let us consider the case of de Sitter space $a(\eta)=-1 / H \eta$. In this case, by inverting (16), $\eta$ is expressed in terms of $\tau$ and $\tilde{k}$ through:

$$
\eta(\tau, \tilde{k})=-\frac{1}{H \tilde{k}} \frac{\sqrt{-\tau e^{\tau}}}{\sqrt{\beta}}
$$

Using (17), (18) and (19), we then obtain for the mode equation in de Sitter space:

$\tilde{\phi}_{\tilde{k}}^{\prime \prime}+\frac{5+\tau}{2 \tau(1+\tau)} \tilde{\phi}_{\tilde{k}}^{\prime}-\left(\frac{1+15 H^{2} \beta+9 H^{2} \beta \tau}{4 H^{2} \beta \tau}\right) \tilde{\phi}_{\tilde{k}}=0$

The creation "time" (when a mode outgrows the minimum length) now corresponds for all $\tilde{k}$ to $\tau_{c}=-1$. (This is particular to the de Sitter case, where $H$ is a constant.) The time $\tau_{c}$ is still a singular point of (22). However, it is now a regular singular point since the Taylor expansions of the term

$$
\left(\tau-\tau_{c}(\tilde{k})\right)^{2} \cdot\left(\frac{1+15 H^{2} \beta+9 H^{2} \beta \tau}{4 H^{2} \beta \tau}\right)
$$

and the term

$$
\left(\tau-\tau_{c}(\tilde{k})\right) \cdot \frac{5+\tau}{2 \tau(1+\tau)}
$$

\footnotetext{
${ }^{5}$ Note that to distinguish between functions in $\eta$ as opposed to functions in $\tau$, we will write a tilde on top of functions in $\tau$, so $a=a(\eta)$, but $\tilde{a}=\tilde{a}(\tau)$ etc. and especially $\phi_{\tilde{k}}=\phi_{\tilde{k}}(\eta)$ but $\tilde{\phi}_{\tilde{k}}=\tilde{\phi}_{\tilde{k}}(\tau)$. This should not be confused with the tilde on $\tilde{k}$ which was introduced in 4 to denote coordinates in which the Fourier modes decouple.
} 
indeed exist. For later convenience, we shift the $\tau$ variable once more so that $x:=\tau+1$; creation time for all modes is now $x_{c}=0$ :

$$
\tilde{\phi}_{\tilde{k}}^{\prime \prime}+\frac{4+x}{2 x(x-1)} \tilde{\phi}_{\tilde{k}}^{\prime}-\left(\frac{1+6 H^{2} \beta+9 H^{2} \beta x}{4 H^{2} \beta(x-1)}\right) \tilde{\phi}_{\tilde{k}}=0
$$

Having transformed the creation time into a regular singular point, we are now ready to solve the mode equation through the Frobenius method.

\subsection{The Frobenius solutions}

Full details on the Frobenius method are given in Appendix A Using the Frobenius ansatz (see (53) in the Appendix) we obtain from (23):

$$
\begin{aligned}
& \sum_{n=0}^{\infty} a_{n}(n+r)(3-n-r) \cdot x^{n-1} \\
+ & \sum_{n=0}^{\infty} a_{n}(n+r)(n+r-1 / 2) \cdot x^{n} \\
- & \sum_{n=0}^{\infty} a_{n}\left(\frac{1}{4 H^{2} \beta}+3 / 2\right) \cdot x^{n+1} \\
- & 9 / 4 \cdot \sum_{n=0}^{\infty} a_{n} \cdot x^{n+2}=0
\end{aligned}
$$

The first line of (24) yields the indicial equation (52), which here has the solutions $r_{1}=3$ and $r_{2}=0$. The first coefficient $a_{0}$ is an arbitrary normalization constant and we set $a_{0}=1$. Comparing the terms of equal powers in $x$, we then find

$$
\begin{aligned}
a_{1}= & \frac{r(2 r-1)}{2(r+1)(r-2)}, \\
a_{2}= & \frac{1}{4(2+r)(1-r)} \\
& \times\left(\frac{1}{H^{2} \beta}+6-\frac{r(2 r-1)(2 r+1)}{r-2}\right) .
\end{aligned}
$$

From $x^{2}$ on, all terms in (24) contribute to the coefficient, thus we can find a recursion formula for $a_{n}$ for $n \geq 3$ as a function of $a_{n-1}, a_{n-2}$ and $a_{n-3}$ as well as $r$ :

$$
\begin{aligned}
a_{n}(r)= & \frac{1}{(n+r)(n+r-3)} \\
& \times\left(-\frac{9}{4} a_{n-3}-\frac{1}{4} a_{n-2}\left[\frac{1}{H^{2} \beta}+6\right]\right. \\
& \left.+\frac{1}{2} a_{n-1}(n+r-1)(2 n+2 r-3)\right)
\end{aligned}
$$

The first solution to (23) is simply given by inserting $r=r_{1}=3$ in the above expressions.
Since $r_{1}-r_{2}=N=3$ is a positive integer, the solution for $r_{2}=0$ is of the form given by (54) in the Appendix. The constant $A$ can be calculated from $a_{3}$ as

$$
A=\lim _{r \rightarrow 0} r \cdot a_{3}(r)=\frac{1}{8 H^{2} \beta} \cdot a_{0} .
$$

The coefficients $c_{n}(r=0)$ for $n=0,1,2, \ldots$ are related to the $a_{n}$ in (25) by

$$
c_{n}\left(r_{2}=0\right)=\left[\frac{d}{d r}\left(\left(r-r_{2}\right) a_{n}(r)\right)\right]_{r=r_{2}=0} .
$$

The second solution to (23) is then obtained by setting $r=r_{2}=0$. (Equivalently, $A$ and the coefficients $c_{0}$ can be calculated directly by inserting (54) into (24), see Appendix A.) The two independent solutions can be combined to form the exact general solution of (23),

$$
\tilde{\phi}_{\tilde{k}}(x)=C_{1} \tilde{\phi}_{\tilde{k}, 1}(x)+C_{2} \tilde{\phi}_{\tilde{k}, 2}(x) .
$$

It is clear from Frobenius theory that the power series solutions converge for all $x<1$, i.e. all $\tau<0$. Here, $C_{1}$ and $C_{2}$ are complex constants whose choice corresponds to the choice of vacuum. Note that our solutions (28) could be rewritten in terms of the conformal time $\eta$ and would then of course solve equation (8).

\subsection{Wronskian condition}

After the transformation $\eta \rightarrow \tau$ the Wronskian condition (13) takes the form:

$$
\tilde{\nu}(\tau, \tilde{k}) \frac{1}{\eta^{\prime}(\tau, \tilde{k})}\left[\tilde{\phi}_{\tilde{k}}(\tau) \tilde{\phi}_{\tilde{k}}^{\prime *}(\tau)-\tilde{\phi}_{\tilde{k}}^{*}(\tau) \tilde{\phi}_{\tilde{k}}^{\prime}(\tau)\right]=i
$$

Note that while the solution space to the mode equation has two complex and therefore four real dimensions, the Wronskian condition reduces the dimensionality to three real dimensions (since only the imaginary part of (29) is nontrivial).

For example, in the case of de Sitter space and when splitting the constants $C_{1}$ and $C_{2}$ in (28) into their real and imaginary parts, $C_{j}=R_{j}+i K_{j}, j=$ 1,2 , the Wronskian condition at the creation time $\tau_{c}=-1$ can be expressed as:

$$
R_{1} K_{2}-K_{1} R_{2}=\left(\frac{12 H}{\beta^{3 / 2} \tilde{k}^{3}}\right)^{-1} .
$$

\subsection{The generic case}

We have here used a suitable transformation of the time variable in order to turn the creation time into a regular singular point so that then the Frobenius 
method yielded the exact solutions to the mode equation in de Sitter space. In fact, this method, using in all cases the same transformation from conformal time to the $\tau$ variable, can also be applied to more general FRW spacetimes. In particular, we have applied the method to the case of general powerlaw backgrounds. The exact solutions are then only slightly more difficult to calculate, see Appendix [C] Namely, while in the de Sitter case we obtained a recursion formula for the coefficients $a_{n}, c_{n}$ as a function of a limited number of predecessors (namely three), in a general power-law background each coefficient generally depends on all others that precede it. Interestingly, the roots of the indicial equation in the general power-law case turn out to be the same as for the de Sitter case, namely $r_{1}=3, r_{2}=0$. Therefore the behaviour of a mode close to its creation time is qualitatively the same in both the de Sitter and the general power-law case.

Further, it is often useful to employ field redefinitions, for example in order to eliminate friction terms in the mode equation. Our method remains applicable under such field redefinitions. In particular, as shown in [5], the field redefinition $\psi_{\tilde{k}}(\eta)=\sqrt{\nu} \phi_{\tilde{k}}(\eta)$ eliminates the friction term in (8), so that we arrive at an equation of the form $\psi_{\tilde{k}}^{\prime \prime}+\omega_{\tilde{k}}^{2}(\eta) \psi_{\tilde{k}}=0$. Similarly, it is possible to eliminate the friction term in our mode equation (23) in which the creation time is a regular singular point. Thereby, the creation time remains a regular singular point. The corresponding field redefinition and exact solutions are presented in Appendix B

Note that formulating the mode equation in frictionless form allows us not only to obtain the exact solutions through the Frobenius method but also to obtain a second set of solutions using the WKB method in the adiabatic regime. This will provide us with two bases of the solution space. These are of course related by a Bogolyubov transformations and we will calculate it Sec 6

\section{Initial conditions}

Having derived the space of exact solutions to the exact mode equation, it becomes crucial to identify the physical solution in that space, since this then implicitly identifies the vacuum state.

For this purpose, given that the mode equation is of second order, it would appear to suffice to specify suitable initial conditions at the mode's creation time, such as the physical solution's value and derivative. Interestingly, the behaviour of the exact solu- tions towards the creation time reveals that the situation is more complex.

Indeed, the values of $\phi_{\tilde{k}}(\eta)$ and its derivative with respect to conformal time, at creation time are given by:

$$
\begin{aligned}
\phi_{\tilde{k}}\left(\eta_{c}\right) & =C_{2} \\
\phi_{\tilde{k}}^{\prime}\left(\eta_{c}\right) & =-\frac{1}{2 \eta_{c}} C_{2}\left(6+\frac{1}{H^{2} \beta}\right)
\end{aligned}
$$

, This shows that prescribing $\phi_{\tilde{k}}\left(\eta_{c}\right)$ and $\phi_{\tilde{k}}^{\prime}\left(\eta_{c}\right)$ will fix only $C_{2}$ but not $C_{1}$. In fact, at the creation time, it is also not possible to specify a solution by specifying higher order derivatives of $\phi_{\tilde{k}}$ since, as one readily verifies, the behaviour of the exact solutions shows that all these higher derivatives are necessarily divergent. For example, $\phi_{\tilde{k}}^{\prime \prime}\left(\eta=\eta_{c}\right)$ diverges as $\propto \frac{1}{\left(\operatorname{plog}\left(-\beta \tilde{k}^{2} / a^{2}\right)+1\right)}$. Roughly speaking, as we approach creation time, the general solution (28) loses its dependence on $C_{1}$.

While the missing $C_{1}$ has two real dimensions, one of these is fixed by the Wronskian condition. Within the remaining one-parameter family of solutions, a solution can be picked by choosing a phase condition, for example, by imposing that the solution be real at a given time $\eta_{a u x} \neq \eta_{c}$, i.e., $\operatorname{Im}\left(\phi_{\tilde{k}}\left(\eta_{a u x}\right)\right)=0$.

Thus, any mode function can be specified by giving the mode function's value at creation time $\eta_{c}$ (two real parameters) and demanding that the solution be real at an auxiliary time $\eta_{a u x}$ (one parameter). The Wronskian condition constrains the fourth parameter.

\section{Comparison with the results of Easther et al.}

In earlier work, see [7, the irregular singular point of the mode equation (8) was dealt with by truncating a series expansion of the coefficients of the mode equation. For the resulting approximate mode equation ${ }^{6}$

$\phi_{\tilde{k}, \text { approx }}^{\prime \prime}-\frac{1}{2\left(\eta-\eta_{c}\right)} \phi_{\tilde{k}, \text { approx }}^{\prime}+\frac{\mathscr{A}}{\left(\eta-\eta_{c}\right)} \phi_{\tilde{k}, \text { approx }}=0$,

$\eta=\eta_{c}$ is a regular singular point. Here, $\mathscr{A}=\frac{\tilde{k} \sqrt{e}}{4 \sqrt{\beta} H}(1+$ $\left.6 \beta H^{2}\right)$. The solution space of the approximate mode

${ }^{6}$ In 7 a different notation for this equation was chosen; apart from replacing the field $\phi_{k}$ by $u_{k}=\phi_{k} / a^{2}$, also the variable $y$ was introduced by setting $\eta=\eta_{c}(1-y)$, and all expressions were re-written in terms of $y$. 
equation (33) was found to be spanned by

$$
F(\eta)=\left(\frac{\sqrt{\mathscr{A}}}{2}+i \mathscr{A} \sqrt{\eta-\eta_{c}}\right) \exp \left(-2 i \sqrt{\mathscr{A}\left(\eta-\eta_{c}\right)}\right)
$$

and its complex conjugate, so that the general solution is given by the linear combination:

$$
\phi_{\tilde{k}, \text { approx }}=\mathscr{C}_{1} F(\eta)+\mathscr{C}_{2} F^{*}(\eta)
$$

Since we have found in Sec 4.2 the exact solutions (28) to the exact mode equation (8), we can now compare them with the solutions to the approximate mode equation that were obtained in [7].

To compare the behaviour of the exact and the approximate solution close to the creation time, we take the first and second $\eta$ derivatives of $F(\eta)$ and evaluate at creation time, which gives

$$
\begin{aligned}
& \phi_{\tilde{k}, \text { approx }}\left(\eta=\eta_{c}\right)=\frac{\sqrt{\mathscr{A}}}{2}\left(\mathscr{C}_{1}+\mathscr{C}_{2}\right), \\
& \phi_{\tilde{k}, \text { approx }}^{\prime}\left(\eta=\eta_{c}\right)=\mathscr{A}^{3 / 2}\left(\mathscr{C}_{1}+\mathscr{C}_{2}\right) .
\end{aligned}
$$

We encounter the same behaviour we observed in the case of the exact solution $\phi_{\tilde{k}}(\eta)$ : the function and its first derivative become proportional to another for $\eta \rightarrow \eta_{c}$ and the proportionality factor is the same as in (31) and (32), namely $2 \mathscr{A}=\frac{\tilde{k} \sqrt{e}}{2 \sqrt{\beta} H}\left(1+6 \beta H^{2}\right)$. Thus, also the solutions to the approximate mode equation show that posing initial conditions on $\phi$ and its derivative at $\eta_{c}$ does not suffice to specify a solution.

Further, the second derivative of the solutions to the approximate mode equation diverges for $\eta \rightarrow \eta_{c}$, namely as $F^{\prime \prime}(\eta) \propto \frac{1}{\sqrt{\eta-\eta_{c}}}$. In comparison, the second $\eta$-derivative of the exact solution $\phi_{\tilde{k}}(\eta)$ at creation time diverges as $\propto\left(\operatorname{plog}\left(-\beta \tilde{k}^{2} H^{2} \eta^{2}\right)+1\right)^{-1}=$ $\frac{\sqrt{-\eta_{c}}}{2} \frac{1}{\sqrt{\eta-\eta_{c}}}+\mathscr{O}(1)$ (using the series expansion of the plog function). Thus, the solutions to the approximate mode equation also properly reproduce the leading divergence for $\eta \rightarrow \eta_{c}$.

We note that the next to leading orders are different: the approximate equation has a regular singular point at creation time which meant that the Frobenius method could be used to derive two power series solutions. The roots of the indicial equation are $(0,3 / 2)$, unlike the roots $(0,3)$ of the exact mode equation. This means that the solutions to the approximate mode equation are of the form: $\phi_{\tilde{k}, \text { approx }, 1}=$ $\sum a_{n}\left(\eta-\eta_{c}\right)^{n}$ and $\phi_{\tilde{k}, \text { approx }, 2}=\sum c_{n}\left(\eta-\eta_{c}\right)^{n+3 / 2}$ The absence of the logarithm in these functions implies that their higher derivatives exhibit a different divergent behaviour than that of the solutions to the exact mode equation.

Related to this, it is clear that the approximate mode equation does not conserve the Wronskian of the exact mode equation. Instead, it conserves the expression $\left|\mathscr{C}_{1}\right|^{2}-\left|\mathscr{C}_{2}\right|^{2}$. Indeed, the Wronskian of the exact mode equation, (13), for the solutions to the approximate mode equation is given by

$$
\mathscr{W}(\eta)=2 \nu(\eta, \tilde{k}) \mathscr{A}^{5 / 2} \sqrt{\eta-\eta_{c}}\left(\left|\mathscr{C}_{1}\right|^{2}-\left|\mathscr{C}_{2}\right|^{2}\right),
$$

where $\nu(\eta, \tilde{k})$, given by (17), has a nontrivial time dependence. Using again the series expansion around $\eta_{c}$, we find that:

$$
\frac{1}{\operatorname{plog}\left(-\beta \tilde{k}^{2} H^{2} \eta^{2}\right)+1}=\frac{\sqrt{-\eta_{c}}}{2} \frac{1}{\sqrt{\eta-\eta_{c}}}+\mathscr{O}(1)
$$

Thus, at $\eta_{c}$, it is possible to enforce the exact Wronskian condition on the solutions to the approximate mode equation, though the Wronskian will be conserved only to first order in $\sqrt{\eta-\eta_{c}}$. This shows that if a solution to the approximate mode equation is to be matched to the numerical evolution of the exact mode equation, as was done in [7, then this match needs to be performed at a time $\eta_{\text {aux }}$ which is as close as possible to the creation time. We note, however, that as our exact solutions will show below, there is limit to how close $\eta_{a u x}$ can be chosen to the creation time, because of unavoidable numerical instabilities.

In [7, a particular choice of initial conditions was suggested, based on a formal similarity between certain solutions to the approximate equation and the solutions that correspond to the Bunch-Davies vacuum in inflation without a cutoff. The argument is that, if the "friction" term in the approximate mode equation (33) could be ignored, then it would be of the form $\phi_{k}^{\prime \prime}+\omega_{k}^{2}(\eta) \phi_{k}=0$ with $\omega_{k}=\sqrt{\frac{\mathscr{A}}{\left(\eta-\eta_{c}\right)}}$. In the region where the adiabaticity condition $\left|\frac{\omega_{\bar{k}}^{\prime}(\eta)}{\omega_{\hat{k}}^{2}(\eta)}\right| \ll 1$ is satisfied, this equation has two approximate solutions of the WKB form:

$$
\begin{aligned}
\phi_{\tilde{k}, \text { approx }}^{\mp}(\eta)= & \left(\frac{\eta-\eta_{c}}{4 \mathscr{A}}\right)^{1 / 4} \\
& \times \exp \left( \pm 2 i \sqrt{\mathscr{A}\left(\eta-\eta_{c}\right)}\right) .
\end{aligned}
$$

Even though one cannot actually ignore the "friction" term in the approximate mode equation because the period close to $\eta_{c}$ is not adiabatic, it is suggestive that these would-be WKB type functions possess the same oscillatory behaviour as Easther et al.'s solution to their approximate mode equation.

In formal analogy to choosing the Bunch-Davies vacuum solution, Easther et al. therefore choose $\mathscr{C}_{2}=$ 
0 so that their preferred physical solution to their approximate mode equation reads ${ }^{7}$

$$
\begin{aligned}
\phi_{\tilde{k}, \text { approx }}= & \mathscr{C}_{1}\left(\frac{\sqrt{\mathscr{A}}}{2}+i \mathscr{A} \sqrt{\eta-\eta_{c}}\right) \\
& \times \exp \left(-2 i \sqrt{\mathscr{A}\left(\eta-\eta_{c}\right)}\right) .
\end{aligned}
$$

The real and imaginary part of $\mathscr{C}_{1}$ are related via the Wronskian condition. The remaining arbitrariness is merely the freedom to choose an overall phase, and can be fixed by demanding, for example, that $\phi_{\tilde{k}, \text { approx }}$ is real at some auxiliary time $\eta_{\text {aux }}$.

Let us now investigate which exact solution to the exact mode equation Easther et al.'s choice corresponds to. Normally, in the case of second order wave equations, in order to match two solutions at any arbitrary point it is sufficient to set these solutions and their first derivatives equal at that point. We would obviously like to match Easther et al.'s function to one of our exact solutions to the exact equation at the creation time. However, such a match-up at creation time itself does here not suffice to use Easther et al.'s choice of mode function to uniquely pick out an exact solution to the exact mode equation. This is because, as we saw earlier, at the creation time $\eta=\eta_{c}$, the value and the first derivative of the solutions are not independent (but are instead a fixed multiple of another).

We therefore studied matching-up the mode function chosen by Easther et al. with an exact solution by setting the functions and their derivatives equal at times $\eta_{m}>\eta_{c}$. We were indeed able to reproduce the observation made in [7] that at times considerably later than $\eta_{c}$ the mode with $\mathscr{C}_{2}=0$ shows close to adiabatic evolution with small characteristic oscillations. This match-up is very delicate, however, and we can now see the underlying reasons.

First, the match cannot be improved by choosing earlier and earlier match-up times $\eta_{m}$ because the mode function's amplitude and derivative loose their independence for $\eta_{m} \rightarrow \eta_{c}$, leading to numerical instability of the match-up.

Second, the match also cannot be improved by choosing late matching times $\eta_{m}$ because the simplified mode equation obeyed by $\phi_{\tilde{k}, \text { approx }}(\eta)$ is close to the precise mode equation only for early $\eta$. Indeed, recall that the function chosen by Easther et al. conserves the Wronskian of the exact mode equation only very close to $\eta_{c}$.

Given that there is no ideal match-up procedure, one may alternatively follow a strategy already out-

${ }^{7}$ Note that even though this solution resembles that of an adiabatic vacuum, it is not because the adiabaticity condition with $\omega_{k}=\sqrt{\frac{\mathscr{A}}{\left(\eta-\eta_{c}\right)}}$ is not satisfied. lined in Sec5 the amplitudes of the two solutions are matched at creation time, which fixes $C_{2}$. The information about $C_{1}$ is then obtained by enforcing the Wronskian condition and by choosing the overall phase such that the mode function is, for example, real at some auxiliary time. We observed again a notable dependence on the arbitrary auxiliary time $\eta_{\text {aux }}$ : it must be chosen not too late after creation time because the approximate mode equation that the function of Easther et al. obeys is close to the exact mode equation only in the vicinity of $\eta_{c}$, but neither must $\eta_{\text {aux }}$ be chosen too early because close to $\eta_{c}$ the exact solution is entirely dominated by one of its dimensions in the solution space and information about the contribution of the other dimension is numerically increasingly difficult to extract.

\section{Comparison with the WKB solution}

The starting point of our present analysis was the mode equation (8), which is analogous to that of a damped harmonic oscillator. We expressed (8) in the $\tau$-variable to obtain (22), which we were then able to solve by means of the Frobenius method. As we show in Appendix B a suitable field redefinition brings (22) into a frictionless form,

$$
\tilde{\psi}_{\tilde{k}}^{\prime \prime}+\tilde{\omega}^{2}(\tau) \tilde{\psi}_{\tilde{k}}=0
$$

see (57), where

$$
\begin{aligned}
\tilde{\omega}^{2}(\tau)= & -\frac{1}{16 H^{2} \beta \tau^{2}(\tau+1)^{2}} \times\left(36 \beta \tau^{4} H^{2}\right. \\
& +132 \beta \tau^{3} H^{2}+153 \beta \tau^{2} H^{2}+30 \beta \tau H^{2} \\
& \left.+5 \beta H^{2}+4 \tau^{3}+8 \tau^{2}+4 \tau\right) .
\end{aligned}
$$

Also this mode equation can be solved with the Frobenius method. We write the general solution in the form

$$
\tilde{\psi}_{\tilde{k}}(\tau)=D_{1} \tilde{\psi}_{\tilde{k}, 1}(\tau)+D_{2} \tilde{\psi}_{\tilde{k}, 2}(\tau),
$$

see (59) in Appendix B

The advantage of the frictionless formulation is that in the adiabatic range of $\tau$, i.e, where $\left|\frac{\tilde{\omega}^{\prime}(\tau)}{\tilde{\omega}^{2}(\tau)}\right| \ll 1$ is fulfilled, (57) will have a set of solutions of the WKB form, namely

$$
\tilde{\Psi}_{\tilde{k}}^{ \pm}(\tau)=\frac{1}{\sqrt{2 \tilde{\omega}(\tau)}} \exp \left(\mp i \int_{\tau_{i}}^{\tau} \tilde{\omega}\left(\tau^{\prime}\right) d \tau^{\prime}\right) .
$$

A general solution to (57) in the adiabatic regime is then given by $\tilde{\Psi}_{\tilde{k}}(\tau)=\lambda_{-} \tilde{\Psi}_{\tilde{k}}^{-}(\tau)+\lambda_{+} \tilde{\Psi}_{\tilde{k}}^{+}(\tau)$. Note 
that the Wronskian condition, when evaluated at $\tau_{c}=$ -1 , imposes:

$$
\left|\lambda_{+}\right|^{2}-\left|\lambda_{-}\right|^{2}=\frac{\tilde{k}^{3} \beta^{3 / 2}}{2 H}
$$

In the adiabatic regime, the exact solutions and the WKB solutions must be related through a Bogolyubov transformation. The WKB solutions, 43), suggest a special choice of the vacuum state, namely the adiabatic vacuum characterized by $\lambda_{-}=0$. The Bogolyubov transformation will then tell us which combination of the exact solutions $\tilde{\psi}_{\tilde{k}, 1}(\tau)$ and $\tilde{\psi}_{\tilde{k}, 2}(\tau)$ this choice corresponds to, i.e., it will determine the coefficients $D_{1}, D_{2}$ in (42).

For the purpose of plotting the properties of the exact solutions, their Frobenius power series expansion, (59), needs to be truncated at some finite order $N$. We established that choosing any $N>30$ suffices to ensure that the plot of the truncated function is valid well into the adiabatic regime.

In order to find the Bogolyubov transformation between the two sets of solutions, it is sufficient to match the value and derivative of the adiabatic solution to an exact solution at some arbitrary time $\tau_{a d}$ in the adiabatic regime:

$$
\begin{aligned}
& D_{1} \tilde{\psi}_{\tilde{k}, 1}\left(\tau_{a d}\right)+D_{2} \tilde{\psi}_{\tilde{k}, 2}\left(\tau_{a d}\right) \\
& \quad=\lambda_{-} \tilde{\Psi}_{\tilde{k}}^{-}\left(\tau_{a d}\right)+\lambda_{+} \tilde{\Psi}_{\tilde{k}}^{+}\left(\tau_{a d}\right) \\
& D_{1} \tilde{\psi}_{\tilde{k}, 1}^{\prime}\left(\tau_{a d}\right)+D_{2} \tilde{\psi}_{\tilde{k}, 2}^{\prime}\left(\tau_{a d}\right) \\
& \quad=\lambda_{-} \tilde{\Psi}_{\tilde{k}}^{\prime}\left(\tau_{a d}\right)+\lambda_{+} \tilde{\Psi}_{\tilde{k}}^{\prime+}\left(\tau_{a d}\right)
\end{aligned}
$$

We verified that the result of the match-up is indeed independent of the match-up time within the adiabatic regime.

\section{Adiabatic vacuum}

We are particularly interested in that mode function $\tilde{\psi}_{\tilde{k}}(\tau)$ which corresponds to the adiabatic vacuum (to zeroth order) in the adiabatic regime. This choice of vacuum is defined by $\lambda_{-}=0$ since in the adiabatic limit only the modes of positive frequency are present. From the match-up of our two sets of solutions we then have

$$
\begin{aligned}
& D_{1}=-\frac{1}{3}\left(\tilde{\Psi}_{\tilde{k}}^{+} \tilde{\psi}_{\tilde{k}, 2}^{\prime}-\tilde{\psi}_{\tilde{k}, 2} \tilde{\Psi}_{\tilde{k}}^{\prime+}\right) \cdot \lambda_{+}, \\
& D_{2}=\tilde{\psi}_{\tilde{k}, 2}^{\prime-1} \cdot\left(\lambda_{+} \tilde{\Psi}_{\tilde{k}}^{\prime+}-D_{1} \tilde{\psi}_{\tilde{k}, 1}^{\prime}\right) .
\end{aligned}
$$

(Here, the fields are understood to be evaluated at the arbitrary time $\tau_{a d}$.) The resulting values of $D_{1}$ and $D_{2}$ are several orders of magnitude apart and not obviously special, in the sense that they do not suggest a particular mathematical criterion that would single out this initial condition and therefore the vacuum in a way that would not rely on the adiabatic expansion. We noticed however, that numerically, $D_{1}$ and $D_{2}$ appear to be at close to 90 degrees when viewed as vectors in the complex plane. In the Outlook we will comment on the potential significance for the choice of vacuum of the existence of an orthonormal basis that is canonical in the solution space.

\section{Deviations from the adiabatic vacuum}

We can now turn this line of argument around and use the knowledge of the exact solutions to bridge the gap between the initial behaviour right at the creation time and the behaviour of the modes in the adiabatic regime. This means that we can precisely link the initial behaviour to the one at horizon crossing which then determines the size and shape of potentially observable effects in the CMB.

Concretely, starting with a certain linear combination of the Frobenius solutions (that may be set by quantum gravity), how close to the adiabatic vacuum will this particular solution be during the adiabatic phase? To this end, we will derive an expression for $\lambda_{-}$, which measures the deviation from the positive frequency adiabatic solution as a function of $D_{1}, D_{2}$. From the match-up (45) we find

$$
\begin{aligned}
\lambda_{-} & =-i\left(D_{1}\left(\tilde{\psi}_{\tilde{k}, 1}^{\prime} \tilde{\Psi}_{\tilde{k}}^{+}-\tilde{\Psi}_{\tilde{k}}^{+\prime} \tilde{\psi}_{\tilde{k}, 1}\right)\right. \\
& \left.+D_{2}\left(\tilde{\psi}_{\tilde{k}, 2}^{\prime} \tilde{\Psi}_{\tilde{k}}^{+}-\tilde{\Psi}_{\tilde{k}}^{+\prime} \tilde{\psi}_{\tilde{k}, 2}\right)\right) .
\end{aligned}
$$

So far, $\lambda_{-}$depends on the real and imaginary parts of $D_{1}$ and $D_{2}$, i.e. on four real parameters:

$$
\begin{aligned}
& D_{1}=S_{1}+i L_{1} \\
& D_{2}=S_{2}+i L_{2}
\end{aligned}
$$

Only two of these parameters are independent, however, due to the Wronskian condition

$$
S_{1} L_{2}-L_{1} S_{2}=\left(\frac{12 H}{\beta^{3 / 2} \tilde{k}^{3}}\right)^{-1},
$$

see Appendix [B] and due to the arbitrariness of the overall phase, which allows us to choose for example $D_{1}$ real. $\left|\lambda_{-}\right|^{2}$ as a function of these remaining two parameters measures the non-adiabaticity of the vacuum in terms of the initial behaviour of the physical mode function. Its minimum is zero and denotes the adiabatic vacuum. 


\section{Behaviour of physical quanti- ties near creation time}

So far, we have parameterized the initial behaviour of modes and used our exact solutions to link it to the mode's behaviour at horizon crossing. In order to better understand what might determine the initial behaviour of modes let us now use our exact solutions to investigate the behaviour of physical quantities such as the mode's field uncertainties and its Hamiltonian close to the mode's creation time.

\subsection{Breakdown of the particle picture}

The Hamiltonian expressed through creation and annihilation operators was given in (14). We see that the nondiagonal terms of the Hamiltonian, i.e., the terms $a_{\tilde{k}} a_{-\tilde{k}}$ and their complex conjugate are proportional to

$$
\mathscr{D}(\eta, \tilde{k})=\frac{\nu}{2} \phi_{-\tilde{k}}^{\prime} \phi_{\tilde{k}}^{\prime}+\frac{\nu}{2}\left(\mu-9\left(\frac{a^{\prime}}{a}\right)^{2}\right) \phi_{-\tilde{k}} \phi_{\tilde{k}} .
$$

The condition $\mathscr{D}(\eta, \tilde{k})=0$ can be rewritten as

$$
\frac{\left(\phi_{\tilde{k}}^{\prime}(\eta)\right)^{2}}{\left(\phi_{\tilde{k}}(\eta)\right)^{2}}=9\left(\frac{a^{\prime}(\eta)}{a(\eta)}\right)^{2}-\mu(\eta, \tilde{k}) .
$$

It is clear that at any finite time $\eta>\eta_{c}$, this equation can be fulfilled, i.e., the Hamiltonian can be made diagonal in the Fock basis, namely by a suitable choice of mode function $\phi_{\tilde{k}}$. For the case of the creation time $\eta_{c}$ itself, however, let us recall from Sec 5 that all mode functions $\phi_{\tilde{k}}$ are proportional to their derivative with a fixed proportionality constant, see (31) and (32). Therefore, (48) cannot be fulfilled at the creation time $\eta_{c}$. This means that whatever mechanism determines the initial behaviour of modes cannot be described in terms of a Fock basis and a corresponding particle picture.

\subsection{Initial field fluctuations}

It has been proposed, see [25], that Planck scale physics might imply that when modes are created they appear in a state that minimizes the product of the fluctuations of the mode's field $\hat{\phi}_{\tilde{k}}(\eta)$ and its conjugate momentum field $\hat{\pi}_{\tilde{k}}(\eta)$ so that the product of the uncertainties reads:

$$
\Delta \hat{\phi}_{\tilde{k}}\left(\eta_{c}\right) \cdot \Delta \hat{\pi}_{\tilde{k}}\left(\eta_{c}\right)=\frac{1}{2}
$$

In our case here, we find that at all times $\eta>\eta_{c}$ :

$$
\begin{aligned}
\Delta \hat{\phi}_{\tilde{k}}(\eta) & =\left|\phi_{\tilde{k}}(\eta)\right| \\
\Delta \hat{\pi}_{\tilde{k}}(\eta) & =\nu(\eta, \tilde{k})\left|\phi_{\tilde{k}}^{\prime}(\eta)-3 \frac{a^{\prime}(\eta)}{a(\eta)} \phi_{\tilde{k}}(\eta)\right|
\end{aligned}
$$

Let us now recall, see Sec5 that while $\phi_{\tilde{k}}$ and its derivative assume finite values for $\eta \rightarrow \eta_{c}$, the term $\nu(\eta, \tilde{k})$ diverges $\propto\left(\operatorname{plog}\left(-\beta \tilde{k}^{2} / a^{2}\right)+1\right)^{-1}$. On one hand, this means that the criterion of [25] for specifying the vacuum state is here not applicable. On the other hand, it is perhaps not surprising that, while the modes are created with field fluctuations $\Delta \hat{\phi}_{\tilde{k}}$ of finite size, their momentum field fluctuations $\Delta \hat{\pi}_{\tilde{k}}$ are divergent at the moment of mode creation itself.

\section{Ground state energy}

Since the Hamiltonian of a mode is quadratic in the field $\pi_{\tilde{k}}$, it is plausible that the divergence of the momentum field's fluctuations $\Delta \hat{\pi}_{\tilde{k}}$ at $\eta_{c}$ should imply a divergence of the expectation value of the mode's Hamiltonian at $\eta_{c}$. To this end, let us consider the total ground state energy at some time $\eta$ :

$$
\begin{aligned}
& E_{v a c}(\eta)=\int_{\tilde{k}^{2}<\frac{a^{2}}{e \beta}} d^{3} \tilde{k} \quad \rho_{v a c, \tilde{k}}(\eta) \\
& =\int_{\tilde{k}^{2}<\frac{a^{2}}{e \beta}} d^{3} \tilde{k}\left[\frac{\nu}{2}\left|\phi_{\tilde{k}}^{\prime}\right|^{2}\right. \\
& \left.+\frac{\nu}{2}\left(\mu-9\left(\frac{a^{\prime}}{a}\right)^{2}\right)\left|\phi_{\tilde{k}}\right|^{2}\right]
\end{aligned}
$$

Recall that the integration ranges over only those modes whose wavelength at time $\eta$ exceeds the cutoff length. At a given mode's creation time its operators $a_{\tilde{k}}$ and $a_{\tilde{k}}^{\dagger}$, first enter the total Hamiltonian, which means that the mode then first contributes to both ground state and dynamical energy. In particular, in an expanding universe, the total Hamiltonian continually picks up new ground state energy as it picks up new modes.

After inserting $\mu(\eta, \tilde{k})$ and $\nu(\eta, \tilde{k})$ into (15) and making use of the fact that we know the exact behaviour of the solutions $\phi_{\tilde{k}}(\eta)$ to the mode equation, we now indeed find a divergence of each mode's contribution to the total ground state energy at the mode's creation time. Concretely, straightforward calculation shows that each mode's Hamiltonian con- 
tains three types of divergent terms:

$$
\begin{aligned}
& \propto \ln \left(\operatorname{plog}\left(-\beta \tilde{k}^{2} / a^{2}\right)+1\right) \\
& \propto 1 /\left(\operatorname{plog}\left(-\beta \tilde{k}^{2} / a^{2}\right)+1\right) \\
& \propto 1 /\left(\operatorname{plog}\left(-\beta \tilde{k}^{2} / a^{2}\right)+1\right)^{3}
\end{aligned}
$$

We obtain a simplified representation of the energetic behaviour of each mode by changing variables from the pair $(\eta, \tilde{k})$ to the new pair $(\tau, \tilde{k})$, and taking into account that the integration measure in (50) transforms as

$$
d^{3} \tilde{k}=4 \pi \tilde{k}^{2} d \tilde{k}=2 \pi \tilde{k}^{3} \frac{(1+\tau)}{\tau} d \tau .
$$

In these variables, each mode's ground sate energy contains only one divergent term, which behaves as $1 /(\tau+1)^{2}$ for $\tau \rightarrow-1$. We can now consider the behaviour of the entity that is of physical significance, namely the integrated ground state energy of all modes.

Here, we notice that the accumulation of ground state energy through mode creation is offset to some extent by the fact that each individual mode's contribution to the total ground state energy $\rho_{v a c, \tilde{k}}(\eta)$, i.e., roughly speaking its $\hbar \omega_{\tilde{k}} / 2$, continually diminishes as its wavelength expands, i.e., as its $\omega_{\tilde{k}}$ decreases.

Whether or not there is a net generating of ground state energy through the expansion depends crucially on the scale factor function $a(\eta)$ of the background spacetime. In the case of the background spacetime that we have considered so far, namely de Sitter space, we know that this calculation can only yield a constant result, since in its mode equation the dependence on $\tilde{k}$ can be eliminated, see (22).

It should be very interesting, however, to carry out the integration of the total ground state energy, at least numerically, for the case of a more general background spacetime, such as the case of power-law inflation. As already mentioned, we have calculated the exact solutions to the exact mode equation in the power-law case, see Appendix [C] The balance of the continual creation of ground state energy and its continual dilution through expansion is nontrivial in any model of quantum field theory in the sub-Planckian regime, including those of generalized dispersion relations. This is of interest because if the vacuum energy generation is not fully offset by its dilution then this would imply a vacuum instability and therefore a potential starting mechanism for inflation.

\section{Summary and outlook}

We considered quantum field theory in the sub-Planckian regime, by which we mean the regime of length scales larger than but close to the Planck (or other cutoff-) length. In the case of an expanding background spacetime the independent degrees of freedom of the QFT, i.e., the field oscillators given by the comoving modes, are of particular interest. This is because as the comoving modes' proper wavelength increases, new comoving modes must continually enter the QFT description of the sub-Planckian regime. A key problem is to identify in which state the comoving modes first enter the QFT description of the sub-Planckian regime and how they evolve through the sub-Planckian regime into the regime where ordinary QFT holds.

To this end, we here followed up on a concrete model for quantum field theory in the sub-Planckian regime. In this model a type of natural UV cutoff is implemented that has been motivated from general quantum gravity arguments, namely the presence of a finite minimum uncertainty in positions. Previous work had found that in this model the initial phase of a mode's evolution is described by a mode equation with an intriguing but difficult to handle singular point at each mode's starting time.

Here, we improved on the existing numerical and semi-analytical solutions by calculating the set of exact solutions to the precise mode equation for the cases of de Sitter space and power-law inflation. In both cases the initial singularity yielded for the roots of the indicial equation the values $(0,3)$. This showed that the qualitative behaviour that can be read off from of our exact solutions around the initial singularity is not dependent on the precise dynamics of the scale factor. This in turn meant that we were able to focus most of our calculations on the simpler case of de Sitter expansion.

Having found the exact solution space, we studied possibilities for identifying the physical mode solution within that solution space and therefore for identifying the initial vacuum. The vacuum determines the mode's late time behaviour at horizon crossing and therefore the type and magnitude of potentially observable effects in the CMB.

Clearly, within any model for QFT in the subPlanckian regime, it is nontrivial to find a reliable method for identifying the physical mode solution. In particular, given any natural UV cutoff it is of course no longer possible to identify a mode's vacuum as having started out essentially as the Minkowski vacuum on the basis that the mode would have had arbitrarily short wavelength in the distant enough past. 
Within the model of QFT in the sub-Planckian regime that we studied here, we found that indeed also any approach that is based on instantaneous Hamiltonian diagonalization in a Fock basis must run into difficulties. The reason is that, as we were able to establish using the exact solutions, each mode's Hamiltonian enters the total Hamiltonian such that it is at the mode's creation time not diagonalizable by means of any Fock representation.

Similarly, the properties of the exact solutions to the mode equation showed that the criterion for identifying the physical mode function by minimizing the field uncertainty relation at mode creation time cannot be applied here unchanged: we found that the field modes themselves are created with finite fluctuations. The canonically conjugate momentum field's fluctuations are, however, divergent at creation time itself, which is plausible given that the momentum field generates changes in the field.

By studying the behaviour of the exact solutions close to the modes' creation time we traced the underlying mathematical reason for why it is difficult to give physical criteria that could reliably single out the physical mode solution. Namely, we found that even though the mode equation is second order in time, at creation time a mode solution cannot be specified as usual by giving its amplitude and derivative. This is because, at the creation time, the amplitude and derivative of all solutions are proportional with the same proportionality constant.

This shows that in any model for QFT in the sub-Planckian regime the physical criteria for specifying the mode function and vacuum may need to be adapted to unexpected mathematical behaviour. Here, for example, it is possible to specify every choice of mode solution by specifying its amplitude at creation time, by using the Wronskian condition and by also using the freedom of overall phase to set the amplitude real at some arbitrary time other than the mode's creation time.

Of course it should be most interesting to try to calculate directly from candidate quantum gravity theories, such as loop quantum gravity and string theory, the mode solution, i.e., to find the state in which these theories predict new comoving modes to enter the sub-Planckian regime in which the framework of QFT is valid. It would be most satisfying then if the fixing of the initial state of the new modes could then be rephrased within QFT in terms of a suitable boundary action along the lines of [26, 27]. Within the model of QFT in the sub-Planckian regime that we studied here, boundary terms have been considered in 28 and those results are likely to be useful for this purpose.
Independent of which initial condition is to be chosen, our exact solutions can be used to straightforwardly calculate from any initial condition the behaviour of the corresponding mode function at horizon crossing. In other words, the explicit solution space provides an explicit bridge between the nontrivial initial conditions that may be set by Planck scale physics and the predictions for their potential impact in the CMB.

We close with a gedanken experiment that indicates that Planck scale physics may not necessarily set initial conditions for modes that enter the subPlanckian regime during expansion, as this may violate unitarity: consider a hypothetical background spacetime that repeatedly contracts and expands, so that comoving modes repeatedly enter and leave the sub-Planckian regime of validity of QFT. In a contracting phase, when a comoving mode's wavelength drops below the cutoff length, the field operators of that mode drop out of the QFT's Hamiltonian. This means that the further evolution of that mode is frozen, at least as far as the QFT is concerned. Whatever excitation or particles that mode might have had are then unaccessible within the framework of QFT because the quantum field simply no longer contains operators that act nontrivially on the dimensions of that mode's Hilbert space.

Thus, for all practical purposes any matter in such a comoving mode would be disappearing as if behind a Planck "horizon". This would be the case until in a subsequent expansion phase the comoving mode's wavelength again exceeds the cutoff length. Then also the Hamiltonian resumes a nontrivial action on that mode's part of the Hilbert space. As far as the framework of QFT is concerned, if the mode froze while in an excited state it will re-emerge in the same excited state during expansion: the evolution is unitary and no information was $\operatorname{lost}^{8}$. It is possible, of course, that the full theory of quantum gravity will show that there are operators which act on those frozen modes' dimensions in the Hilbert space so that in a re-expanding phase, when the modes re-enter the description by the framework of QFT, they do so with certain fixed initial conditions. Clearly, in this case unitarity would be hard to maintain in a cycle of expansions and contractions. If, however, Planck scale physics were not to enforce a fixed initial condition on modes, then the question remains unanswered in which state comoving modes are created when they

\footnotetext{
${ }^{8}$ It should be worth investigating if a similar Hilbert space mechanism to the one discussed here may freeze degrees of freedom that fall into a black hole, to thaw them in the final evaporation of the black hole, thus preserving unitarity without having the frozen degrees of freedom unduly gravitate.
} 
first emerge, during the very first expansion.

This leads to the question whether in the solution space to the mode equation there exists any distinguished or canonical solution that might therefore be the preferred physical solution for modes that emerge for the first time, i.e., the question is if the mathematics singles out a preferred vacuum state. This amounts to asking if there exists a canonical splitting of the solution space that could improve on the useful but at best approximate splitting of the solution space into positive and negative frequency solutions during periods of adiabatic evolution. Here we notice that, equivalently, the question is whether there is a canonical split of the solution space into what in an adiabatic phase would be the sine and cosine basis solutions to the mode equation (from which one would then obtain positive and negative frequency solutions straightforwardly). Because of the peculiar singularity at the mode creation time this is indeed possible: recall that there exists a distinguished dimension of the solution space, consisting of the real-valued mode solutions that vanish at creation time. This induces a canonical $\mathrm{ON}$ basis in the two-dimensional solution space that could be viewed as the generalizations of the sine and cosine functions. It will be difficult to orthonormalize these functions in practice because the exact solution's power series is needed in an integration over all time while the power series is slow to converge at late times. Nevertheless, it will be very interesting to investigate how close the so-defined positive frequency mode solution would be to the adiabatic vacuum in the adiabatic phase.

\section{Acknowledgements}

This work was partially supported by PREA, CFI, OIT and the Canada Research Chairs program of the National Science and Engineering Research Council of Canada. LL acknowledges support by the International Council for Canadian Studies during the early phase of this work and current support by the DAAD.

\section{References}

[1] J. Martin and R. H. Brandenberger, Phys. Rev. D63, 123501 (2001), hep-th/0005209.

[2] R. H. Brandenberger and J. Martin, Mod. Phys. Lett. A16, 999 (2001), astro-ph/0005432.

[3] J. C. Niemeyer, Phys. Rev. D63, 123502 (2001), astro-ph/0005533.

[4] A. Kempf, Phys. Rev. D63, 083514 (2001), astro-ph/0009209.
[5] A. Kempf and J. C. Niemeyer, Phys. Rev. D64, 103501 (2001), astro-ph/0103225.

[6] A. A. Starobinsky, Pisma Zh. Eksp. Teor. Fiz. 73, 415 (2001), astro-ph/0104043.

[7] R. Easther, B. R. Greene, W. H. Kinney, and G. Shiu, Phys. Rev. D64, 103502 (2001), hepth/0104102.

[8] L. Hui and W. H. Kinney, Phys. Rev. D65, 103507 (2002), astro-ph/0109107.

[9] R. Easther, B. R. Greene, W. H. Kinney, and G. Shiu, Phys. Rev. D67, 063508 (2003), hepth/0110226.

[10] R. Easther, B. R. Greene, W. H. Kinney, and G. Shiu, Phys. Rev. D66, 023518 (2002), hepth/0204129.

[11] J. Martin and C. Ringeval, JCAP 0501, 007 (2005), hep-ph/0405249.

[12] J. C. Niemeyer and R. Parentani, Phys. Rev. D64, 101301 (2001), astro-ph/0101451.

[13] T. Tanaka, (2000), astro-ph/0012431.

[14] A. Kempf, On the structure of space-time at the Planck scale, in 36th Course: From the Planck Length to the Hubble Radius, Erice, Italy, 29 Aug - 7 Sep 1998, edited by A. Zichichi, p. 613, World Scientific, Singapore, 2000.

[15] D. J. Gross and P. F. Mende, Nucl. Phys. B303, 407 (1988)

[16] D. Amati, M. Ciafaloni, and G. Veneziano, Phys. Lett. B216, 41 (1989).

[17] L. J. Garay, Int. J. Mod. Phys. A10, 145 (1995), gr-qc/9403008.

[18] G. Amelino-Camelia, J. R. Ellis, N. E. Mavromatos, and D. V. Nanopoulos, Mod. Phys. Lett. A12, 2029 (1997), hep-th/9701144.

[19] E. Witten, Phys. Today 49, 24 (1996).

[20] D. J. Gross and P. F. Mende, Nucl. Phys. B303, 407 (1988).

[21] A. Kempf, J. Math. Phys. 35, 4483 (1994), hepth/9311147.

[22] A. Kempf, J. Phys. A30, 2093 (1997), hepth/9604045.

[23] A. Kempf, Phys. Rev. Lett. 85, 2873 (2000), hep-th/9905114. 
[24] A. Kempf, Phys. Rev. Lett. 92, 221301 (2004), gr-qc/0310035.

[25] U. H. Danielsson, Phys. Rev. D66, 023511 (2002), hep-th/0203198.

[26] K. Schalm, G. Shiu, and J. P. van der Schaar, JHEP 04, 076 (2004), hep-th/0401164.

[27] B. R. Greene, K. Schalm, G. Shiu, and J. P. van der Schaar, JCAP 0502, 001 (2005), hepth/0411217.

[28] A. Ashoorioon, A. Kempf, and R. B. Mann, Phys. Rev. D71, 023503 (2005), astro$\mathrm{ph} / 0410139$.

\section{A The Frobenius method}

Consider a differential equation of the form

$x^{2} \cdot y^{\prime \prime}(x)+x \cdot[x \cdot p(x)] \cdot y^{\prime}(x)+\left[x^{2} \cdot q(x)\right] \cdot y(x)=0$,

where $x=0$ is a regular singular point, i.e., the coefficients have convergent Taylor series expansions

$$
\begin{aligned}
x \cdot p(x) & =\sum_{n=0}^{\infty} p_{n} x^{n} \\
\text { and } x^{2} \cdot q(x) & =\sum_{n=0}^{\infty} q_{n} x^{n}
\end{aligned}
$$

in some interval $|x|<\rho$. The Frobenius method states that then there exists a so-called indicial equation:

$$
r \cdot(r-1)+p_{0} \cdot r+q_{0}=0
$$

Let $r_{1}$ and $r_{2}$ be the two roots of this equation, with $r_{1} \geq r_{2}$, if $r_{1}$ and $r_{2}$ are real. Then (51) has either on the interval $-\rho<x<0$ or on $0<x<\rho$ one solution of the form

$$
y_{1}(x)=|x|^{r_{1}} \sum_{n=0}^{\infty} a_{n}\left(r_{1}\right) \cdot x^{n} .
$$

In the case where the two roots $r_{1}$ and $r_{2}$ of (52) differ by an integer (so that $r_{1}-r_{2}=N$ ), the second solution takes the form

$$
y_{2}(x)=A \cdot y_{1}(x) \cdot \ln |x|+|x|^{r_{2}} \sum_{n=0}^{\infty} c_{n}\left(r_{2}\right) \cdot x^{n} .
$$

The constant $A$ as well as the $a_{n}\left(r_{1}\right), c_{n}\left(r_{2}\right)$ can be determined by inserting each of the solutions into the equation (51) ${ }^{9}$. The solutions converge at least for $|x|<\rho$ and define a function which is analytic around $x=0$.

\footnotetext{
${ }^{9}$ Once having found the coefficients $a_{n}$ of the first solution, $A$ and the $c_{n}$ can also be calculated from them; see Sec 4.2 and Appendix B
}

\section{B Exact solution to the friction- less mode equation}

As shown in [5], the field redefinition $\psi_{\tilde{k}}(\eta)=\sqrt{\nu} \phi_{\tilde{k}}(\eta)$ allows to eliminate the friction term in (8), so that we arrive at an equation of the form $\psi_{\tilde{k}}^{\prime \prime}+\omega_{\tilde{k}}^{2}(\eta) \psi_{\tilde{k}}=0$ with

$$
\begin{aligned}
\omega_{\tilde{k}}^{2}(\eta)= & \mu-6\left(\frac{a^{\prime}}{a}\right)^{2}+\left(\frac{\nu^{\prime}}{2 \nu}\right)^{2}-3\left(\frac{a^{\prime} \nu^{\prime}}{a \nu}\right) \\
& -3\left(\frac{a^{\prime \prime}}{a}\right)-\left(\frac{\nu^{\prime \prime}}{2 \nu}\right) .
\end{aligned}
$$

The Wronskian condition (13) also simplifies to

$$
\psi_{\tilde{k}}(\eta) \psi_{\tilde{k}}^{\prime *}(\eta)-\psi_{\tilde{k}}^{*}(\eta) \psi_{\tilde{k}}^{\prime}(\eta)=i .
$$

Here we determine the corresponding field redefinition for the case of the mode equation formulated in the $\tau$-variable, namely equation (22).

Making the ansatz $\tilde{\phi}_{\tilde{k}}(\tau)=F(\tau) \tilde{\psi}_{\tilde{k}}(\tau)$ and inserting into equation (22), we find $F(\tau)=\frac{\tau+1}{\tau^{5 / 4}}$. Recall that the range for the variable $\tau$ is $-1 \ldots 0$. Therefore, for later convenience, we take a factor of $\sqrt{i}$ out of $F(\tau)$ and write the field redefinition in $\tau$ as

$$
\tilde{\phi}_{\tilde{k}}(\tau)=-\frac{\tau+1}{\sqrt{i}(-\tau)^{5 / 4}} \tilde{\psi}_{\tilde{k}}(\tau)
$$

After the transformation (55), the equation of motion for the new field $\tilde{\psi}_{\tilde{k}}(\tau)$ is $\tilde{\psi}_{\tilde{k}}^{\prime \prime}+\tilde{\omega}_{\tilde{k}}^{2}(\tau) \tilde{\psi}_{\tilde{k}}=0$ with

$$
\begin{aligned}
\tilde{\omega}_{\tilde{k}}^{2}(\tau)= & \tilde{\mu} \eta^{\prime 2}-3\left(\frac{\tilde{a}^{\prime \prime}}{\tilde{a}}\right)-6\left(\frac{\tilde{a}^{\prime}}{\tilde{a}}\right)^{2}-3 \frac{\tilde{a}^{\prime} \tilde{\nu}^{\prime}}{\tilde{a} \tilde{\nu}} \\
& +3 \frac{\tilde{a}^{\prime}}{\tilde{a}} \frac{\eta^{\prime \prime}}{\eta^{\prime}}-\frac{5+\tau}{4 \tau(1+\tau)} \cdot\left(\frac{\tilde{\nu}^{\prime}}{\tilde{\nu}}-\frac{\eta^{\prime \prime}}{\eta^{\prime}}\right) \\
& +\frac{5(\tau+9)}{16 \tau^{2}(1+\tau)} .
\end{aligned}
$$

Substituting $x:=\tau+1$, the mode equation we need to solve reads (note that the creation time now corresponds to $\left.x_{c}=0\right)$ :

$$
\begin{gathered}
\tilde{\psi}_{\tilde{k}}^{\prime \prime}-\frac{1}{16 \beta H^{2} x^{2}(x-1)^{2}} \cdot\left(36 \beta H^{2} x^{4}\right. \\
-12 \beta H^{2} x^{3}-27 \beta H^{2} x^{2}-24 \beta H^{2} x \\
\left.+32 \beta H^{2}+4 x^{3}-4 x^{2}\right) \tilde{\psi}_{k}=0
\end{gathered}
$$

(The prime now denotes $\partial / \partial \tau$.) Since this equation is of the form (51) with $\tilde{p}(x)=0$ and the desired behaviour for $x^{2} \cdot \tilde{q}(x)$, we can use the Frobenius method to solve it. 


\section{Application of the Frobenius method}

Our ansatz is $\tilde{\psi}_{\tilde{k}}(x)=x^{s} \sum_{n=0}^{\infty} b_{n}(s) \cdot x^{n}$, which, sorted by powers of $x$, leads to

$$
\begin{aligned}
& \sum_{n=0}^{\infty} b_{n}[(n+s)(n+s-1)-2] x^{n} \\
+ & \sum_{n=0}^{\infty} b_{n}\left[-2(n+s)(n+s-1)+\frac{3}{2}\right] x^{n+1} \\
+ & \sum_{n=0}^{\infty} b_{n}\left[(n+s)(n+s-1)+\frac{27}{16}+\frac{1}{4 H^{2} \beta}\right] x^{n+2} \\
+ & \sum_{n=0}^{\infty} b_{n}\left[\frac{3}{4}-\frac{1}{4 H^{2} \beta}\right] x^{n+3} \\
- & \sum_{n=0}^{\infty} b_{n} \frac{9}{4} x^{n+4}=0 .
\end{aligned}
$$

The indicial equation in this case, found from the coefficient of $x^{0}$, reads

$$
s(s-1)-2=0,
$$

with the two roots $s_{1}=2$ and $s_{2}=-1$. Note that again we find an integer difference between the two indicial roots, $s_{1}-s_{2}=N=3$. Therefore the first solution is of the form (53) with $s_{1}=2$, while the second solution is of the form (54) with $s_{2}=-1$. We set the arbitrary normalization constant $b_{0}=1$.

We further find that

$$
\begin{aligned}
b_{1}= & \frac{4 s(s-1)-3}{2(s(s+1)-2)} \\
b_{2}= & \frac{1}{(s+1)(s+2)-2} \\
& \times\left(\frac{(4 s(s-1)-3)(4 s(s+1)-3)}{4(s(s+1)-2)}\right. \\
b_{3}= & \frac{1}{(s+2)(s+3)-2} \\
& \quad \times\left[\left(2(2+s)(s+1)-\frac{3}{2}\right) b_{2}\right. \\
& \quad-\left(s(s+1)+\frac{27}{16}+\frac{1}{4 H^{2} \beta}\right) b_{1}
\end{aligned}
$$

For $n \geq 4$, we can establish a recursion formula for each coefficient $b_{n}$ as a function of its four predeces- sors $^{10}$ and $s$ :

$$
\begin{aligned}
b_{n}(s)= & \frac{1}{(n+s)(n+s-1)-2} \\
& \times\left[\left(2(n+s-1)(n+s-2)-\frac{3}{2}\right) b_{n-1}\right. \\
& \quad-((n+s-2)(n+s-3) \\
& \left.\quad+\frac{27}{16}+\frac{1}{4 H^{2} \beta}\right) b_{n-2} \\
& +\left(\frac{1}{4 H^{2} \beta}-\frac{3}{4}\right) b_{n-3} \\
& \left.+\frac{9}{4} b_{n-4}\right]
\end{aligned}
$$

To determine the $b_{n}$ for the first solution, simply insert $s=s_{1}=2$ in the expressions above.

The second solution is of the form

$$
\tilde{\psi}_{\tilde{k}, 2}(x)=B \cdot \tilde{\psi}_{\tilde{k}, 1}(x) \cdot \ln x+x^{s_{2}} \sum_{n=0}^{\infty} d_{n}\left(s_{2}\right) \cdot x^{n}
$$

with $s_{2}=-1$. Using our results for the first solution $\tilde{\psi}_{\tilde{k}, 1}$, we find $(N=3)$

$$
B=\lim _{s \rightarrow-1}(s+1) \cdot b_{N}(s)=\frac{1}{8 H^{2} \beta}
$$

and

$$
d_{n}(s=-1)=\left[\frac{d}{d s}\left((s+1) b_{n}(s)\right)\right]_{s=s_{2}=-1} .
$$

Again, the general solution to (57) is given by a linear combination,

$$
\tilde{\psi}_{\tilde{k}}(x)=D_{1} \tilde{\psi}_{\tilde{k}, 1}(x)+D_{2} \tilde{\psi}_{\tilde{k}, 2}(x),
$$

where $D_{1}$ and $D_{2}$ are complex constants.

\section{Wronskian condition}

To find the corresponding Wronskian condition, we plug the field redefinition into (29). This leads to

$$
\begin{gathered}
\tilde{\nu}(\tau, \tilde{k}) \frac{1}{\eta^{\prime}}\left(\frac{\tau+1}{(-\tau)^{5 / 4}}\right)^{2} \\
\times\left[\tilde{\psi}_{\tilde{k}}(\tau) \tilde{\psi}_{\tilde{k}}^{\prime *}(\tau)-\tilde{\psi}_{\tilde{k}}^{*}(\tau) \tilde{\psi}_{\tilde{k}}^{\prime}(\tau)\right]=i .
\end{gathered}
$$

Splitting the constants $D_{1}$ and $D_{2}$ into their real and imaginary parts,

$$
\begin{aligned}
& D_{1}=S_{1}+i L_{1} \quad \text { and } \\
& D_{2}=S_{2}+i L_{2},
\end{aligned}
$$

\footnotetext{
${ }^{10}$ Note that here the regression depth is greater by one than in the case of the solution to 22 .
} 
imposing (60) at the creation time $x_{c}=0$ leads to $x_{c}=0$ :

the condition:

$$
S_{1} L_{2}-L_{1} S_{2}=\left(\frac{12 H}{\beta^{3 / 2} \tilde{k}^{3}}\right)^{-1}
$$

\section{Exact solution for the gen- eral power-law case}

The scale factor in a general power-law background reads

$$
a(\eta)=\left(\frac{\eta}{\eta_{0}}\right)^{q}
$$

so that the inverse transformation $\eta \rightarrow \tau$ (compare (16)) is given by

$$
\eta=\eta_{0}\left(-\frac{\beta \tilde{k}^{2}}{\tau e^{\tau}}\right)^{\frac{1}{2 q}}
$$

It can be shown that the exact mode equation (20) written for the power-law case still satisfies the necessary conditions for applying the Frobenius method. That is, the creation time $x_{c}=0$ is a regular singular point of the explicit transformed mode equation

$$
\begin{array}{r}
x(x-1)^{2} \tilde{\phi}_{\tilde{k}}^{\prime \prime} \\
+\left(\left(\frac{1}{2}+\frac{1}{2 q}\right) x^{3}-\frac{1}{2 q} x^{2}+\frac{3}{2} x-2\right) \tilde{\phi}_{\tilde{k}}^{\prime} \\
+\tilde{G}\left(q, \eta_{0} ; x\right) \tilde{\phi}_{\tilde{k}}=0
\end{array}
$$

where we have used the abbreviation

$$
\begin{aligned}
\tilde{G}\left(q, \eta_{0} ; x\right)= & \left(\frac{3}{4 q}-\frac{3}{2}\right) x^{3}+\frac{3}{4} x^{2} \\
& +\left[\frac{3}{2}+\frac{\tilde{k}^{2} \eta_{0}^{2}}{4 q^{2} e^{x-1}}\right. \\
& \left.\times\left(-\frac{\beta \tilde{k}^{2}}{(x-1) e^{x-1}}\right)^{\frac{1}{q}}\right] x .
\end{aligned}
$$

We now work with the Frobenius ansatz

$$
\tilde{\phi}_{\tilde{k}}\left(q, \eta_{0} ; x\right)=\sum_{n=0}^{\infty} f_{n}\left(q, \eta_{0}, u\right) x^{n+u} .
$$

The $f_{n}\left(q, \eta_{0}, u\right)$ can be determined by inserting (66) into (64) and sorting by powers of $x$. Since, however, in general the coefficient $\tilde{G}\left(q, \eta_{0}, x\right)$ need not be polynomial, we calculate its Taylor expansion around

$$
\begin{aligned}
\tilde{G}\left(q, \eta_{0}, x\right) \approx & \tilde{G}\left(q, \eta_{0}, 0\right) \\
& +\left(\frac{\tilde{k}^{2} \eta_{0}^{2} e}{4 q^{2}}\left(\beta \tilde{k}^{2} e\right)^{\frac{1}{q}}+\frac{3}{2}\right) x \\
& +\left(-\frac{\tilde{k}^{2} \eta_{0}^{2} e}{4 q^{2}}\left(\beta \tilde{k}^{2} e\right)^{\frac{1}{q}}+\frac{3}{4}\right) x^{2} \\
& +\sum_{m=3}^{\infty} \frac{1}{m !}\left(\frac{\partial^{m} \tilde{G}\left(q, \eta_{0}, x\right)}{\partial x^{m}}\right)_{x=0} x^{m}
\end{aligned}
$$

Proceeding exactly as before (see Sec 4 and Appendix A), we find the same roots of the indicial equation as in the de Sitter case, namely $u_{1}=3, u_{2}=0$. Therefore, the structure of the solutions (i.e., one a mere polynomial, (53), the other containing a logarithmic term, (54)) is the same in de Sitter case as well as in all power-law backgrounds.

The explicit expressions for the $f_{n}\left(q, \eta_{0}, u\right), n=$ 1,2,3 found from inserting of the Frobenius ansatz are (where we set $f_{0}=1$ ):

$$
\begin{aligned}
f_{1}(u)= & -\frac{u(7-4 u)}{2(1+u)(u-2)} \\
f_{2}\left(q, \eta_{0}, u\right)= & -\frac{1}{(u+2)(u-1)}\left[u\left(u-1-\frac{1}{2 q}\right)\right. \\
& -u \frac{(7-4 u)(3 / 2-2 u)}{2(u-2)} \\
f_{3}\left(q, \eta_{0}, u\right)= & \left.-\frac{1}{(3+u) u}+\frac{\tilde{k}^{2} \eta_{c}^{2} e}{4 q^{2}}\right] \\
& \times\left\{(2+u)\left(-2 u-\frac{1}{2}\right) \cdot f_{2}\left(q, \eta_{0}, u\right)\right. \\
& +\left[(u+1)\left(u-\frac{1}{2 q}\right)\right. \\
& \left.+\frac{3}{2}+\frac{\tilde{k}^{2} \eta_{c}^{2} e}{4 q^{2}}\right] \cdot f_{1}(u) \\
& \left.+\left(\frac{3}{4}+u\left(\frac{1}{2}+\frac{1}{2 q}\right)-\frac{\tilde{k}^{2} \eta_{c}{ }^{2} e}{4 q^{2}}\right)\right\}
\end{aligned}
$$

Here we used $\eta_{c}$ as an abbreviation; in the power-law case $\eta_{c}=\eta_{0}\left(\beta \tilde{k}^{2} e\right)^{\frac{1}{2 q}}$

In the general power-law background case the coefficient $f_{n}\left(q, \eta_{0}, u\right)$ for $n \geq 4$ depends on all the coefficients preceding it. Explicitly, the recursion formula 
reads:

$$
\begin{aligned}
& f_{n}\left(q, \eta_{0}, u\right)=-\frac{1}{(n+u)(n+u-3)} \\
& \times\{(n+u-1) \\
& \times\left(\frac{3}{2}-2(n+u-2)\right) \cdot f_{n-1} \\
& +\left[(n+u-2)\left(n+u-3-\frac{1}{2 q}\right)\right. \\
& \left.+\frac{3}{2}+\frac{\tilde{k}^{2} \eta_{c}^{2} e}{4 q^{2}}\right] \cdot f_{n-2} \\
& +\left[(n+u-3)\left(\frac{1}{2}+\frac{1}{2 q}\right)\right. \\
& \left.+\frac{3}{4}-\frac{\tilde{k}^{2} \eta_{c}^{2} e}{4 q^{2}}\right] \cdot f_{n-3} \\
& +\sum_{m=4}^{m=n} \frac{1}{(m-1) !} \\
& \left.\times\left(\frac{\partial^{m-1} \tilde{G}\left(q, \eta_{0}, x\right)}{\partial x^{m-1}}\right)_{x=0} \cdot f_{n-m}\right\}
\end{aligned}
$$

The first solution $\left(u_{1}=3\right)$ in the general power-law background then reads

$$
\tilde{\phi}_{\tilde{k}, 1}\left(q, \eta_{0} ; x\right)=x^{3} \sum_{n=0}^{\infty} f_{n}\left(q, \eta_{0}, u=3\right) x^{n} .
$$

The second solution has the form

$$
\begin{aligned}
\tilde{\phi}_{\tilde{k}, 2}\left(q, \eta_{0} ; x\right)= & F \cdot \tilde{\phi}_{\tilde{k}, 1}\left(q, \eta_{0} ; x\right) \cdot \ln x \\
& +\sum_{n=0}^{\infty} g_{n}\left(q, \eta_{0}, u_{2}=0\right) x^{n},
\end{aligned}
$$

where the constant $F$ can be calculated to read

$$
F=\frac{\tilde{k}^{2} \eta_{c}^{2} e}{8 q^{2}} .
$$

The coefficients $g_{n}\left(q, \eta_{0}, u=0\right)$ of the second power series are related to the $f_{n}\left(q, \eta_{0}, u\right)$ by:

$$
g_{n}\left(q, \eta_{0}, u_{2}=0\right)=\left[\frac{d}{d u}\left(u \cdot f_{n}\left(q, \eta_{0}, u\right)\right)\right]_{u=u_{2}=0}
$$

The general solution for the power-law background case is given by the linear combination of the two solutions:

$$
\tilde{\phi}_{\tilde{k}}\left(q, \eta_{0} ; x\right)=E_{1} \tilde{\phi}_{\tilde{k}, 1}\left(q, \eta_{0} ; x\right)+E_{2} \tilde{\phi}_{\tilde{k}, 2}\left(q, \eta_{0} ; x\right)
$$

$E_{1}, E_{2}$ are complex constants, constrained by the Wronskian condition, which in the power-law case becomes

$$
\begin{aligned}
- & \frac{2 q}{\eta_{0}} \frac{e^{\tau / 2} \tau^{3}}{\beta^{2} \tilde{k}^{4}(1+\tau)^{2}}\left(-\frac{\tau e^{\tau}}{\beta \tilde{k}^{2}}\right)^{\frac{1}{2 q}} \\
& \times\left[\tilde{\phi}_{\tilde{k}}(\tau) \tilde{\phi}_{\tilde{k}}^{* \prime}(\tau)-\tilde{\phi}_{\tilde{k}}^{*}(\tau) \tilde{\phi}_{\tilde{k}}^{\prime}(\tau)\right]=i .
\end{aligned}
$$

Using a Taylor expansion of the prefactor,

$$
\begin{array}{r}
-\frac{2 q}{\eta_{0}} \frac{e^{\tau / 2} \tau^{3}}{\beta^{2} \tilde{k}^{4}(1+\tau)^{2}}\left(-\frac{\tau e^{\tau}}{\beta \tilde{k}^{2}}\right)^{\frac{1}{2 q}} \approx \\
\frac{2 q\left(\frac{1}{e \beta \tilde{k}^{2}}\right)^{1 / 2 q}}{\sqrt{e} \beta^{2} \tilde{k}^{4} \eta_{0}} \cdot \frac{1}{x^{2}}-\frac{5 q\left(\frac{1}{e \beta \tilde{k}^{2}}\right)^{1 / 2 q}}{\sqrt{e} \beta^{2} \tilde{k}^{4} \eta_{0}} \cdot \frac{1}{x}+\ldots,
\end{array}
$$

and splitting $E_{1,2}$ into real and imaginary part, $E_{j}=$ $T_{j}+i M_{j}, j=1,2$, the condition we find from evaluating (67) at the creation time $x_{c}=0$ reads:

$$
T_{1} M_{2}-M_{1} T_{2}=\frac{\sqrt{e} \beta^{2} \tilde{k}^{4} \eta_{0}}{12 q\left(\frac{1}{e \beta \tilde{k}^{2}}\right)^{1 / 2 q}}
$$

